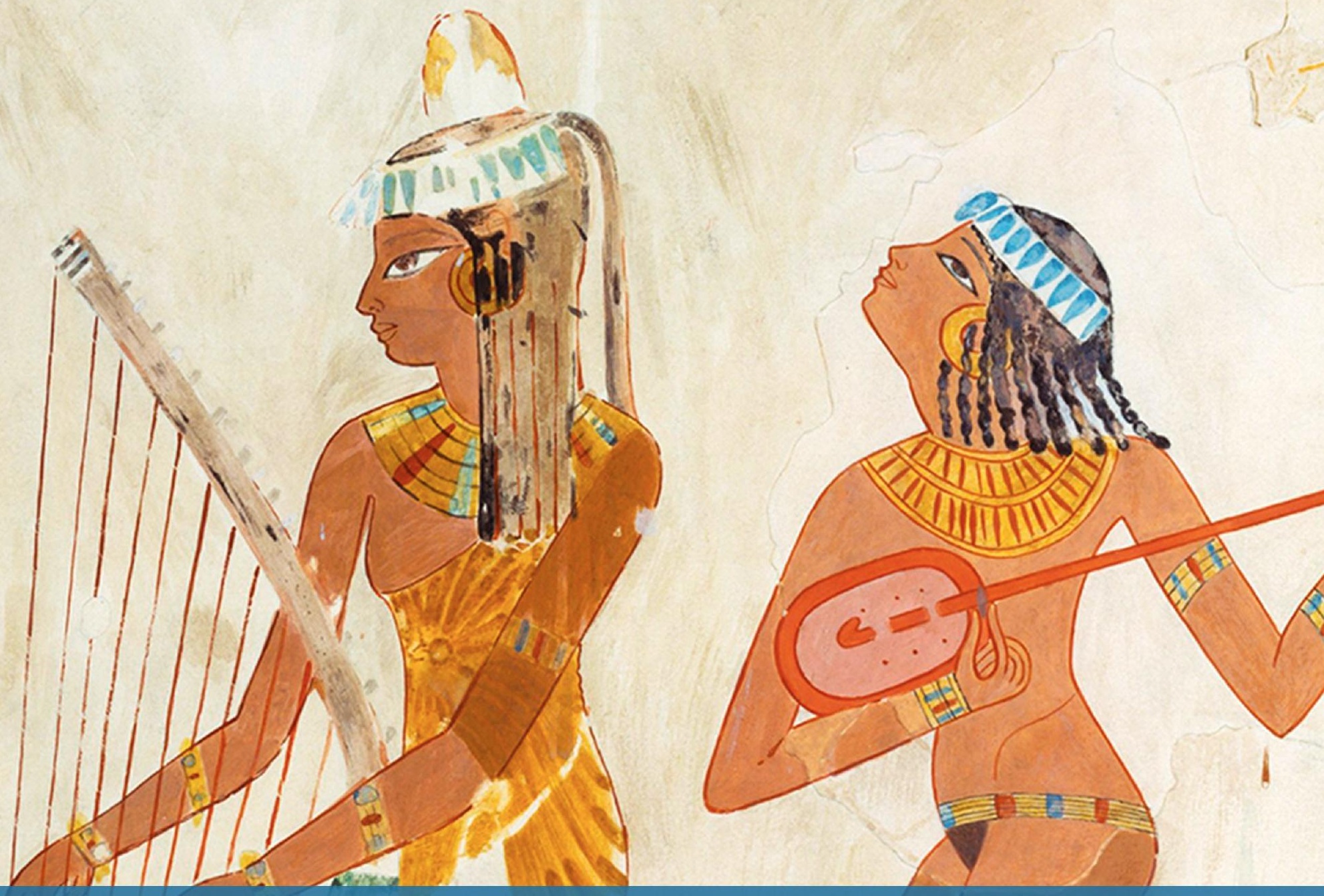

Revista digital de ciencia y Didáctica de la Historia

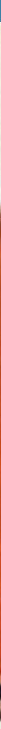




\section{Panta Rei \\ Revista Digital de Ciencia \\ y Didáctica de la Historia}

\section{9}

Revista anual

Fecha de inicio: 1995

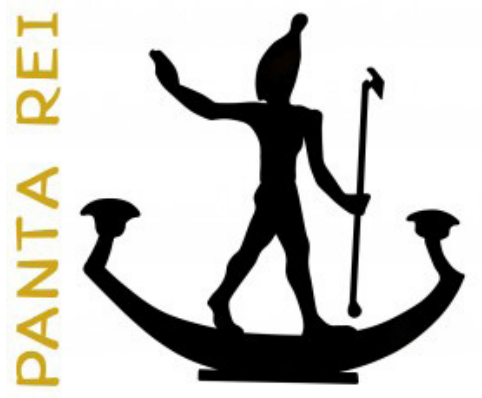

Revista Panta Rei. pantarei@um.es

Edita:

Centro de Estudios del Próximo Oriente y la

Antigüedad Tardía - CEPOAT

Edificio Universitario Saavedra Fajardo.

Universidad de Murcia

C/ Actor Isidoro Máiquez, 9

30007 - MURCIA - ESPAÑA

Teléfono: (+34) 868883890

cepoat@um.es

Web: www.um.es/cepoat/pantarei

Edición 2019

ISSNe: 2386-8864

Responsable de la presente edición: Consejo Editorial Panta Rei.

UNIVERSIDAD DE MURCIA centro de estudios del próximo oriente y la antigüedad tardía

En Portada: Fragmento de pintura de la tumba de Djeserkareseneb (TT38), Tebas. (fotografía del Metropolitan Museum).

Responsables de los textos: Sus autores.

ISSN: 1136-2464

Depósito legal: MU-966-1995 


\section{CONSEJO DE REDACCIÓN}

\section{Coordinador editorial}

Egea Vivancos, Alejandro

[Didáctica de las Ciencias Sociales, UMU]

\section{Editores}

Jiménez Vialás, Helena

[UMU]

López Muñoz, Dámaris

[UJA]

Meseguer Gil, Antonio José

[CEPOAT, UNED]

Sáez Giménez, David Omar

[CEPOAT, UMU]

Sánchez Mondéjar, Celso Miguel

[Patrimonio Inteligente]

\section{Secretaria}

Arias Ferrer, Laura

[Didáctica de las Ciencias Sociales, UMU]

\section{Responsable informático}

Martínez García, José Javier

[CEPOAT, UMU]

\section{Traducción y corrección lingüística}

Martínez Martínez, Cristina

[Sociedad Española de Lenguas Modernas]

Albaladejo Albaladejo, Sara

[ISEN, UMU]

\section{CONSEJO ASESOR}

Adroher Auroux, Andrés María [Arqueología, Universidad de Granada]

Albero Muñoz, M. ${ }^{a}$ del Mar [Historia del Arte, Universidad de Murcia]

Alia Miranda, Francisco [Historia Contemporánea, UCLM]

Arciniega García, Luis [Historia del Arte, Universidad de Valencia]

Barrio Barrio, Juan Antonio [Historia Medieval, Universidad de Alicante]

Castellano i Solé, Núria [Egiptología, Schola Didàctica Activa S.L.]
Chapman, Arthur [History Education, University College of London, Reino Unido]

Cid López, Rosa María [Historia Antigua, Universidad de Oviedo]

Cobacho López, Ángel [Derecho, Universidad de Murcia]

Cuenca López, José María [Didáctica de las Ciencias Sociales, Universidad de Huelva]

Egea Bruno, Pedro M. ${ }^{a}$ [Historia Contemporánea, Universidad de Murcia]

Feijoo Martínez, Santiago [Arqueología, Consorcio Ciudad Monumental de Mérida]

García Atienzar, Gabriel [Prehistoria, Universidad de Alicante]

Ginestí Rosell, Anna [Filología Clásica, Katholische Universität Eichstätt-Ingolstadt]

González Monfort, Neus [Didáctica de las Ciencias Sociales, Universidad Autónoma de Barcelona]

González Soutelo, Silvia [Arqueología, Universidad de Vigo]

Haber Uriarte, María [Prehistoria, Universidad de Murcia]

Hernández de la Fuente, David [Historia Antigua, Universidad Complutense]

Hutson, Scott R. [Anthropology, University of Kentucky, EE UU]

Igual Luis, David [Historia Medieval, UCLM]

Irigoyen López, Antonio [Historia Moderna, Universidad de Murcia]

Jover Maestre, Francisco Javier [Prehistoria, Universidad de Alicante]

Mahony, Simon [Digital Humanities, University College of London, Reino Unido]

Marsilla de Pascual, Francisco Reyes [Técnicas historiográficas, Universidad de Murcia]

Martínez-Burgos García, Palma [Historia del Arte, UCLM]

Mathis, Christian [Didaktik der Geschichte, PH Zürich]

Miralles Maldonado, José Carlos [Filología Clásica, Universidad de Murcia]

Molina Gómez, José Antonio [Historia Antigua, Universidad de Murcia]

Mónica Ghirardi [Historia Moderna, Universidad Nacional de Córdoba, Argentina]

Navarro Espinach, Germán [Historia Medieval, Universidad de Zaragoza]

Noguera Celdrán, José Miguel [Arqueología, Universidad de Murcia]

Ortiz Heras, Manuel [Historia Contemporánea, UCLM]

Panzram, Sabine [Historia Antigua, Universität Hamburg]

Pérez Molina, Miguel Emilio [Filología Clásica, Universidad de Murcia]

Prados Martínez, Fernando [Arqueología, Universidad de Alicante]

Sánchez Ibáñez, Raquel [Didáctica de las Ciencias Sociales, Universidad de Murcia]

Sancho Gómez, Miguel Pablo [Educación, UCAM]

Victoria Moreno, Diego [Historia Contemporánea, UNED]

Vilar García, María José [Historia Contemporánea, Universidad de Murcia]

Vivas Sainz, Inmaculada [Historia del Arte, UNED]

Zamora López, José Ángel [Próximo Oriente Antiguo, CCHS-CSIC] 



\section{Índice}

\section{Artículos}

La imagen de la Prehistoria en el cine y los géneros del cine prehistórico. Un mundo de hombres mono, bikinis y dinosaurios.

Alberto Lombo Montañés.

Trabajo y roles de género durante la Prehistoria. Un estudio sobre su percepción en el alumnado de Historia.

María Pastor Quiles y Daniel Mateo Corredor.

Memoria cultural en el Egipto Faraónico. Algunas reflexiones sobre su origen, función y pervivencia histórica.

Antonio Pérez Largacha.

La representación de la danza dentro de las escenas de banquete de las tumbas tebanas privadas de la XVIII dinastía egipcia.

Miriam Bueno Guardia.

La "Sala del Fresco" de Micenas. Revisión de las interpretaciones del programa iconográfico y nueva lectura en relación a los espacios.

Pelayo Huerta Segovia.

Las advertencias de Quilón y Solón sobre la tiranía de Pisístrato.

Unai Iriarte.

La Virgen de la Leche. Arquetipo de mujer y madre en la pintura del Renacimiento español.

Patricia Castiñeyra Fernández

Innovación didáctica en Historia: un estado de la cuestión en torno a cuatro ejes temáticos.

Diego Luna Delgado.

\section{Reseñas}

Foster, B. R. (2016), The Age of Agade. Inventing empire in ancient Mesopotamia, Londres y Nueva York: Routledge, 438 págs.

Juan Álvarez García.

Alviz Fernández, M. y Hernández de la Fuente, D. (Eds.) (2017). De ópos a limes: el concepto de frontera en el mundo antiguo y su recepción. Madrid: Escolar y Mayo editores. 256 págs.

Ethan Yepes de la Hoz.

Clares Clares, M ${ }^{\mathrm{a}}$ E. (2017). Música y noches de moda. Sociedades, cafés y salones domésticos de Murcia en el siglo XIX. Murcia: Universidad de Murcia, Servicio de Publicaciones. 474 págs.

Elena Micó Terol 195

Goñi Zabalegui, A. (2018). Género y sociedad en el Egipto romano. Una Mirada desde las cartas de mujeres. Oviedo: Ediciones de la Universidad de Oviedo. 360 págs.

Alejandra Izquierdo Perales. 199

Normas de publicación/Publishing rules 



\title{
Foster, B. R. (2016), The Age of Agade. Inventing empire in ancient Mesopotamia, Londres y Nueva York: Routledge, 438 págs.
}

\author{
Para citar esta reseña: Álvarez García, J. (2019). [Reseña del libro The Age of Agade. Inventing \\ empire in ancient Mesopotamia, de Foster, B. R]. Panta Rei. Revista Digital de Ciencia y Didáctica \\ de la Historia, 185-189.
}

ISSNe: 2386-8864

DOI: $10.6018 /$ pantarei/2019/09

Profesor de Asiriología en la Universidad de Yale, Benjamin R. Foster es un gran especialista en estudios sobre el Próximo Oriente antiguo. De entre sus líneas de investigación están la Historia, general, económica y social de Mesopotamia así como también sus estudios sobre literatura mesopotámica contando con trabajos como el galardonado Civilizations of Ancient Iraq (2010) o la obra Before the Muses (última edición de 2005), una antología de la literatura acadia ya considerada una obra clave en los estudios sobre historia intelectual del Próximo Oriente antiguo.

En el presente trabajo, encuadrado en esa línea de investigación más centrada en los estudios históricos, la intención del autor es mostrar las características políticas sociales, económicas y culturales que se desarrollaron en la región de Mesopotamia durante el periodo de Akkad y por qué motivos este periodo fue visto como un referente a lo largo de la Historia posterior del Próximo Oriente durante la antigüedad.

El primer capítulo está dedicado a la historia política de Akkad. Este periodo comienza con la llegada al trono de Kish de Sargón y sus diferentes campañas de conquista, ganándose la fama de gran rey con la que se le recordará en periodos posteriores. No obstante, su hijo y sucesor, Rimush, tendrá que hacer frente a un conjunto de revueltas tras la muerte de su padre; incluso es posible que acabara sus días asesinado. Tras esto, su hermano Manishtushu alcanzó el trono iniciando un programa de conquistas y de consolidación de las mismas. Posteriormente, su hijo Naram-Sin toma las riendas del estado y su reinado constituirá el apogeo del Imperio. Al igual que antecesores suyos tuvo que hacer frente a levantamientos, por lo que es posible que el recuerdo de la represión ejercida contra los sublevados le valiera la fama posterior de rey soberbio. Durante el reinado de Naram-Sin se llevó a cabo un amplio programa constructivo y una serie de reformas administrativas y burocráticas. En política exterior, junto a las conquistas también se desarrolla la diplomacia, con matrimonios dinásticos con reinos fronterizos. Por último, el sucesor de Naram-Sin, Sharkalisharri será el último gran rey de Akkad. Finalmente, las sublevaciones e invasiones exteriores, en un contexto de crisis económica y descontrol territorial, provocarán la caída de este proyecto político.

El autor reserva el capítulo segundo a dar las claves de la sociedad durante el periodo acadio. Seguramente sea el régimen ecológico de la zona norte de Mesopotamia la que de coherencia al territorio original acadio frente a la llanura aluvial del sur, Sumer. Es en esta zona donde se desarrolla (pero no exclusivamente) un sustrato etno-lingüístico acadio. Si en términos jurídicos debemos hablar de dos grupos de población: libres y esclavos, vemos que en términos socioeconómicos estos se diversifican en relación a las propiedades y los medios con los que cuentan. Las relaciones 
entre los miembros de las distintas clases sociales se rigen por redes clientelares y de patronazgo. De esta forma, la administración se organiza como una red de patrones y clientes que tienen en su cúspide al propio rey, seguido por sus familiares más allegados, administradores centrales, provinciales, cultuales, militares y todo el personal administrativo dependiente de ellos.

En el capítulo tres, el autor hace una descripción de los asentamientos acadios y de aquellos centros constatados arqueológicamente desde donde el poder acadio ejercía su autoridad tanto en Mesopotamia como en la periferia. De los diferentes asentamientos se destaca la especial importancia dada por parte de la administración imperial a la explotación económica de sus territorios circundantes así como a su papel estratégico como centros de recepción de materias primas desde la periferia, como Tell Brak, Assur o Susa. Finalmente, el autor reflexiona sobre si se puede calificar al estado acadio como Imperio, afirmando que sí si atendemos al programa de conquistas y control del territorio y a la ideología real que se desarrolla de "dominio universal".

En relación al anterior, en el capítulo cuatro se explican el conjunto de bases económicas y las actividades y trabajos desarrollados en torno a ellas. Las principales actividad económica es la agricultura y la ganadería, donde era fundamental la explotación de la región del sur mesopotámico (Sumer). En cuanto al trabajo, éste estaba basado en trabajadores dependientes a tiempo completo y trabajadores reclutados en épocas muy específicas, todos retribuidos mediante sistemas de raciones. En relación a la ganadería, destacaba la oveja por su lana, la principal materia de transformación y exportación. En lo referente al comercio y transporte, el autor destaca los cursos fluviales como vía principal de comunicación, además de ser fuente de recursos pesqueros. La última parte del capítulo está dedicada al conjunto de actividades de transformación de materias primas en alimento, destacando la molienda y la producción de cerveza.

El siguiente capítulo versa sobre el conjunto de actividades de tipo artesanal/industrial que se desarrollaron en el periodo acadio. Muchas son herederas de épocas anteriores, pero durante ésta podemos apreciar una alta estandarización producto de una mayor concentración de artesanos en talleres reales y una mayor cantidad de bienes gracias a, por un lado, una mayor importación de materias primas y, por otro, una mayor demanda por parte de las élites. Así pues, el autor comienza analizando la producción cerámica; pero destaca sobre todo los trabajos en metal, piedra y madera. alcanzándose una gran maestría técnica en ellas. También hay que destacar los textiles en lana o en piel; así como las artesanías más selectas como la ebanistería o los aceites perfumados.

En lo que respecta al capítulo seis, dedicado a la religión, el autor destaca una serie de innovaciones pese a la gran continuidad en la evolución de la religiosidad mesopotámica. Las divinidades acadias que se incorporan al panteón mesopotámico destacan por ser divinidades celestiales: Shamash, Sin, Ishtar, etc. y por participar en una mitología guerrera. De entre las mayores innovaciones está la deificación de ciertos reyes en vida, como Naram-Sin, junto a la elevación de Ishtar a lo alto del panteón nacional y la política de integración de cultos y divinidades acadias con sumerias. Si bien podemos identificar ciertos templos particulares del periodo acadio, en la mayor parte de los santuarios reina la continuidad. En estos se aprecia la vinculación entre religión y política puesto que son los reyes los que llevan a cabo ritos y realizan ofrendas suntuosas. Entre las formas de piedad colectiva siguen estando las festividades, las cuales carecen de un calendario estandarizado para todo el imperio.

En cuanto al aspecto militar, tratado en el capítulo siete, vemos como los reyes acadios recogen una serie de tradiciones anteriores, como el denominarse elegidos por Enlil para reinar sobre Mesopotamia. Pero, por otra parte, fomentaron el aspecto guerrero del rey y sus capacidades personales como aptitudes necesarias para ejercer la realeza. En lo tocante a la composición y armamento del ejército, vemos que esto no cambia demasiado respecto a periodos anteriores, exceptuando la organización del mismo que es puesto bajo la autoridad de militares profesionales.

El corto capítulo ocho está dedicado al comercio y las diferentes formas de intercambio. Como ya se ha dicho, la llanura mesopotámica carece de una serie de materias primas fundamentales que debían importarse; en época acadia lo que se aprecia es un incremento en dichas importaciones. En torno a la naturaleza de este comercio, la existencia de mercaderes privados que podían estar 
también al servicio de las grandes instituciones, la existencia de medios de pago estandarizados como la plata y la cebada, así como también de tasas, impuestos y precios estipulados indican que la economía real del periodo era plenamente tributaria y no exclusivamente redistributiva.

El capítulo nueve viene a tratar todo lo referente a las artes y a la producción literaria. Aquí podemos apreciar una línea transversal en el arte acadio, la inclinación por representar la ideología real basada en el militarismo, la fuerza, la heroicidad y la especial relación del rey con los dioses. La escultura, el relieve e incluso la glíptica, desarrollan estos temas y en ellas se alcanza una alta perfección técnica considerándose el periodo clásico de la escultura en Mesopotamia. En literatura sobresale la princesa y sacerdotisa Enheduanna. Esta poetisa (primera de la literatura mundial) también sirvió con su obra a la ideología real a través de sus himnos a los dioses y a los reyes. En prosa destacan las inscripciones conmemorativas, y la epistolografía, que adquieren un importante valor literario. En esta producción literaria hay que destacar el uso paralelo del acadio y el sumerio como lenguas eruditas, junto al desarrollo de la música que acompañaba la representación de las composiciones literarias. Por último, la matemática y la cartografía cuentan con una importante presencia asociada a la administración.

Una vez señalada la identidad del arte y la producción intelectual, el autor centra el capítulo diez en definir los valores humanos acadios, en otras palabras, la identidad acadia. En primer lugar a través de ciertos aspectos de la vida cotidiana como el nacimiento, la niñez y la educación, la vida familiar y la casa y la muerte y el funeral. Pero el autor también analiza los sentimientos y las emociones; de las cuales solo tenemos testimonio de las experimentadas por las élites. Aquí el autor comenta como se entendía en el periodo acadio la felicidad y la tristeza, el amor y la sexualidad y el espíritu competitivo entre los miembros de la élite que pugnaban por ascender dentro de la administración imperial.

En el capítulo once, el autor reflexiona sobre la memoria de los reyes de Akkad en periodos posteriores de la historia de Mesopotamia. Si bien algunos de ellos siguieron siendo reverenciados e incluso se mantuvo su culto funerario, otros recibieron el castigo y la deshonra. Sin embargo, en su gran mayoría las estelas de los reyes acadios permanecieron en los santuarios en donde fueron erigidas, siendo copiadas por escribas y eruditos. De hecho, el autor traza una relación entre menciones a los reyes acadios en la literatura profética posterior y los hechos contados en las estelas, por lo que dichos presagios se inspiraban en estas narraciones. Las crónicas posteriores no se olvidaron tampoco de los reyes acadios generándose incluso en torno a ellos una rica literatura épica. Por su parte, el legado acadio se aprecia en los nombres y titulatura de muchos reyes posteriores, queriendo emular la fuerza y poder de sus antecesores.

El último capítulo de la obra consiste en una reflexión sobre los estudios en torno al periodo acadio. Así pues, su presencia en la historiografía sobre el próximo oriente antiguo comienza con el descubrimiento, entre mediados y finales del siglo XIX, de textos e inscripciones que hacían referencia a los reyes acadios. A partir de aquí se sucedieron durante la primera mitad del siglo XX los hallazgos y las interpretaciones sobre quiénes eran y de donde procedían. Y fue a partir de entonces cuando se empezaron a publicar las primeras síntesis. No obstante, no fue hasta el descubrimiento de los archivos de Ebla en 1975 cuando se empezó a contar con un volumen importante de información.

De este modo, Benjamin R. Foster nos ofrece una completa y detallada síntesis del periodo acadio. Podemos ver cómo el denominado Imperio de Akkad hereda una serie de procesos históricos, sociales y económicos que se iniciaron en etapas previas, así como también un conjunto de estructuras políticas e ideológicas que recogen los reyes acadios. Sin embargo, este conjunto de características heredadas se potencian en esta etapa a todos los niveles: una más alta concepción de la realeza, una burocracia estatal más sólida, una explotación de los recursos más intensiva, un deseo de compenetración de las identidades socioculturales que componían Mesopotamia. Una aceleración de procesos que alcanza el apogeo en durante el reinado de Naram-Sin. Se generó así un conjunto de características exclusivas sin las cuales no podríamos explicar la historia posterior. De esto se dieron cuenta incluso los propios antiguos, reteniendo en su memoria a los poderosos 
reyes acadios.

No obstante, el profesor Foster es demasiado optimista al calificar de "Imperio" al proyecto político de los reyes acadios. Esto va más allá de un simple calificativo, puesto que el concepto histórico de "imperio" encierra unas connotaciones ideológicas y unos desarrollos políticos, sociales, económicos y culturales mucho mayores que aquellas a las que llegaron los reyes de Akkad. Sin lugar a dudas este periodo marcó la historia posterior de Mesopotamia en particular y del Próximo Oriente en general, dejando una fuerte impronta en el imaginario colectivo de la región. No obstante, aquellos que defienden la naturaleza imperial del estado acadio, se dejan llevar por las fuentes posteriores que tanto veneraron la tradición de aquellos reyes. Si estudiamos la naturaleza del periodo en su contexto, vemos un alcance limitado del "Imperio" tanto en su plano ideológico como fáctico.

En este sentido, hay que decir que el debate no cosiste en preguntarse si Akkad fue o no un imperio, un error metodológico por el cual se pretende adscribir el hecho a un concepto historiográfico convirtiendo así el concepto y no el hecho en el objeto último de nuestra investigación, perdiendo por tanto el concepto su capacidad de ser herramienta explicativa del hecho histórico. Por este motivo, en primer lugar, debemos preguntarnos, ¿qué es un imperio? Si lo estudiamos desde una perspectiva más amplia, podemos ver que, a lo largo de la historia, el denominador común de todo imperio es su ideología, por lo tanto, no podemos disociar imperio de imperialismo. En este sentido, el profesor Mario Liverani hace una interesante reflexión sobre el concepto "misión imperial" en su recentísima obra, Assiria. La preistoria dell'imperialismo, Bari: Laterza, 2017. Según este concepto, la clave para poder calificar una estructura política de imperio es la necesidad de conquistar, unificar, ordenar y gobernar el mundo, generándose unas estructuras políticas e ideológicas que se derivan de este conjunto de intenciones.

Siguiendo esta norma, no podríamos calificar de imperio al estado que crean los reyes acadios. Para empezar, los afanes de dominio universal de los que hacen gala los reyes acadios no corresponden a una ideología imperialista, sino más bien a una propaganda real propia del periodo por la que desean legitimar su gobierno a través de sus propias cualidades guerreras, heroicas y, en ciertos casos, divinas. Esto se aprecia además en que los reyes de Akkad, una vez unificada Mesopotamia, no dirigen empresas de conquista más allá, sino más bien desarrollan una serie de campañas destinadas a mantener bajo control puntos estratégicos necesarios para el abastecimiento de materias primas. Esto igualmente lo vemos en la producción artística y literaria, encaminada a servir de canal de propaganda de la realeza y no a ser la muestra de la gloria y el poder el supuesto imperio.

En su vertiente más económica, la posición de vanguardia que toma la región de Akkad frente a Sumer no se debió tanto a la política económica activa de los reyes acadios, sino que responde a una dinámica ecológica por la cual los territorios aguas arriba de los dos grandes cauces fluviales tienen ventaja sobre las tierras que hay en la llanura aluvial, cuyas aguas tienden a la salinidad y el estancamiento. Si bien no podemos negar que el dominio acadio sobre toda Mesopotamia y ejercido desde esa región del norte (económicamente más favorable) pudiera acelerar el proceso, tampoco podemos afirmar de ninguna manera que fuera una política consciente de los reyes acadios. En primer lugar, porque es un proceso ecológico que se encuadra en un marco cronológico mucho más amplio, que se inició antes de las conquistas de Sargón de Akkad y que continuará tras la caída del dominio acadio, con la excepción del periodo de gobierno de la III dinastía de Ur y sólo gracias a los ingentes esfuerzos de sus gobernantes por revertir dicho proceso.

Igualmente, en su faceta socio-cultural, no podemos adscribir a los reyes acadios el que el elemento semítico de la sociedad se anteponga al elemento sumerio. Esto tiene un proceso paralelo al ecológico del que hemos hablado anteriormente. Se trata de un proceso etnolingüístico por el cual aquellas lenguas que encuentran facilidades de traducción y reproducción en otras del entorno tienden a perpetuarse. En este sentido, el sumerio, pese a haber sido la lengua en la que se escribieron los primeros textos y constituir la base cultural de los primeros estados de Mesopotamia, no deja de ser un grupo etnolingüístico aislado, sin paralelos en otras lenguas. Por el contrario, el 
acadio, como lengua perteneciente al tronco semítico, encuentra fácil traducción y perpetuación en otras lenguas del entorno, como el eblaíta; por lo tanto, la fluidez de información es mucho mejor entre distintos territorios. Así pues, de forma semejante a lo que se ha comentado sobre el proceso ecológico que se desarrolla en Mesopotamia, el proceso etnolingüístico que favorecía al elemento acadio sobre el sumerio pudo ser acelerado por los reyes acadios, pero no podemos adscribirles a ellos el mérito de tal hecho puesto que continuará en periodos posteriores cuando los reyes acadios ya eran tan sólo un recuerdo y el sumerio quede relegado por completo al papel de lengua erudita.

Así pues, calificar de "Imperio" al estado unificado de Mesopotamia bajo el gobierno de la dinastía de Akkad sería algo erróneo. Pese a esto, no podemos obviar el hecho de que no se trató de un estado territorial más, puesto que se implementan muchas de las estructuras políticas y económicas previas, así como se aceleran muchos de los procesos históricos sin los cuales no podríamos entender la Historia posterior del Próximo Oriente. Por este motivo, Mario Liverani, en la obra conjunta que él mismo edita, Akkad, the first world empire: structure, ideology, traditions, Padova: Sargon, 1993; recurre al término de "red imperial". Según este concepto, el estado acadio no habría cambiado las estructuras políticas y económicas previas, sino que se habría asentado sobre ellas controlando exclusivamente las relaciones entre las mismas, convirtiéndose así el estado central en punto de intersección de dichas estructuras.

\author{
Juan Álvarez García \\ Universidad Autónoma de Madrid
}





\section{Panta Rei}

PANTA REI es una revista digital de investigación orientada a la Historia y otras ciencias afines. Su principal objetivo es la transmisión del conocimiento científico, dando una oportunidad también a los jóvenes investigadores que quieren abrirse camino en el estudio de las ciencias humanas y sociales. Se compone de estudios originales relacionados con la disciplina histórica así como su didáctica y difusión. Las diferentes secciones que componen la revista son: artículos de investigación, entrevistas a profesionales, recensiones de monografías de actualidad y crónicas de congresos o eventos científicos relevantes.

Todos los artículos publicados son objeto de un proceso de revisión a cargo de un mínimo de dos evaluadores, que se consideran expertos en el ámbito temático del artículo propuesto. Nuestro deseo es poder ofrecer unos contenidos rigurosos, de calidad y de interés.

EI CEPOAT (Centro de Estudios del Próximo Oriente y la Antigüedad Tardía de la Universidad de Murcia) es la institución encargada de la coordinación y gestión de la revista, desde donde anualmente se lanzará la convocatoria para aquellos que estén interesados en publicar sus trabajos, siempre relacionados con la Historia, Arqueología, Historia del Arte, Didáctica de la Historia, etc.

PANTA REI is a digital journal focused on History and other sciences related to it. Its main objective is the transmission of scientific knowledge by giving also an opportunity to young researchers who want to make their way in the study of human and social sciences. It is composed by original studies related to History, as well as its didactics and promotion. The different sections of this journal are: research articles, interviews to professionals, recensions on monographs about current issues and reports about congresses or relevant scientific events.

All the articles published are subject to a revision process carried out by a minimum of two reviewers who are considered to be experts in the field of the article proposed. Our wish is to offer rigorous contents with quality and being of interest to the reader.

CEPOAT (Centre of Studies of the Middle East and Late Antiquity of the University of Murcia) is the institution in charge of the coordination and management of this journal. This is the centre from where the call for papers will be launched for all the people interested in publishing their papers, always related to History, Archeology, Art History, Teaching History, etc. 


\section{Normas de Publicación}

El autor se compromete a enviar trabajos originales, que no se encuentren publicados en otras revistas ni en otros idiomas. Así mismo, el mismo artículo no podrá ser presentado en otras revistas mientras dure el proceso de evaluación.

\section{Envío y presentación de originales}

Los artículos se enviarán exclusivamente a través del correo electrónico a la dirección pantarei@um.es. Los textos serán enviados en formato DOC y las imágenes en formato JPEG o TIFF, y con un tamaño mínimo de 2000 px. Éstas no aparecerán incorporadas en el texto, sino enviadas en archivo aparte y correctamente numeradas según su posición en el texto. Junto al trabajo, se rellenará y enviará un documento aparte en el que se especifiquen los datos del autor siguiendo el modelo disponible en la página Web de la revista.

Para la redacción de los trabajos se tendrá en cuenta el Manual de la American Psychological Association, en su sexta edición. La extensión máxima de los trabajos será de 30 páginas. La tipografía será Arial 11, con interlineado sencillo y sin espacio alguno entre párrafos. El texto deberá ir justificado a ambos márgenes y sin sangría en los primeros párrafos. Los márgenes serán de $2,50 \mathrm{~cm}$. En los casos en los que fuera necesario incorporar notas, éstas irán a pie de página, enumeradas consecutivamente, con tipografía Arial 10, interlineado sencillo y justificadas a ambos márgenes.

Una información más detallada se encuentra disponible en la página http://www.um.es/cepoat/ pantarei.

\section{Proceso de valoración y evaluación}

Una vez recibidos los trabajos, la Revista realizará una primera valoración. Si el trabajo enviado se ajusta a las normas de presentación propuestas, la temática es coincidente con la línea editorial de la revista y posee la calidad científica necesaria, será remitido al consejo asesor para una primera evaluación. Si no es así en este primer paso se puede rechazar directamente los documentos que incumplan claramente la línea editorial.

Será el Consejo Asesor quien indique a la revista la originalidad, relevancia, estructura, redacción, aparato bibliográfico, etc. del trabajo enviado y, para ello, se designará a dos revisores expertos externos que evaluarán cada uno de los trabajos, que pueden formar parte (o no) de este Consejo Asesor. La selección de los revisores se ajustará a la temática y características metodológicas del trabajo. El nombre y filiación de los autores serán eliminados del trabajo para su revisión, así como los revisores actuarán de manera anónima y confidencial.

Los revisores deberán rellenar un informe de evaluación que centrará su atención en aspectos tales como características formales, originalidad y novedad de los trabajos, relevancia de las propuestas y los resultados, calidad metodológica y validez científica.

Una vez terminado el proceso se decidirá la aceptación o no de los mismos y su publicación en el número que sea pertinente, así como las modificaciones susceptibles de ser realizadas para su final publicación. Dicha notificación se enviará únicamente por correo electrónico, en un plazo máximo de seis meses. 


\section{Publishing rules}

The author is committed to submit original papers not having been published in other reviews or in other languages. In this way, it is not allowed for the same paper to be presented in other reviews during the evaluation process.

\section{Submission and presentation of originals}

The articles will be exclusively submitted by email to pantarei@um.es. The texts will be submitted in DOC format and the images in JPEG or TIFF format, and with a minimum size of 2000 px. Images will not be integrated in the text but sent in another file and properly numbered according to their position in the text. Attached to the paper, a document will be filled out and sent where the author's data will be specified following the model available on the website.

The sixth edition of the Manual of the American Psychological Association will be taken into account for the writing of the papers. The length of the papers must not exceed 30 pages. Typography will be Arial 11 , with simple line spacing and no space between paragraphs. The text must be justified on both margins without indentation in the first paragraphs. Margins size will be $2.50 \mathrm{~cm}$. Where it could be necessary the incorporation of notes, they will be at the bottom of the page, consecutively numbered with typography Arial 10, simple line spacing and justified on both margins.

More detailed information is available on the website: http://www.um.es/cepoat/pantarei.

\section{Examination and assessment process}

The Journal will submit the papers to a first examination once received. If the paper follows the presentation guidelines, the subject agrees with the editorial line of this journal, and possess the scientific quality required, it will be sent to the advisory council for a first assessment. If not, the documents which clearly fail to complete the editorial line may be rejected straightaway in this first step.

The Advisory Council will indicate the originality, relevance, structure, writing, bibliography, etc. of the text to the journal; for this purpose, two outside experts will be designated to review the papers; these experts can be (or not) part of this Advisory Council. The selection of the experts will adjust to the subject and methodological characteristics of the paper. Name and affiliation of the author will be eliminated from the text for its review, in this way experts will act anonymously and confidentially.

The experts will fill out an assessment report which will focus on aspects such as formal characteristics, originality and novelty of the papers, relevance and results of the proposal, methodological quality and scientific validity.

Once the process is finished, the acceptance or not of the papers and its publication in the corresponding edition will be decided, as well as the modifications that may be done for its final publication. This notification will be sent by email within 6 months maximum. 


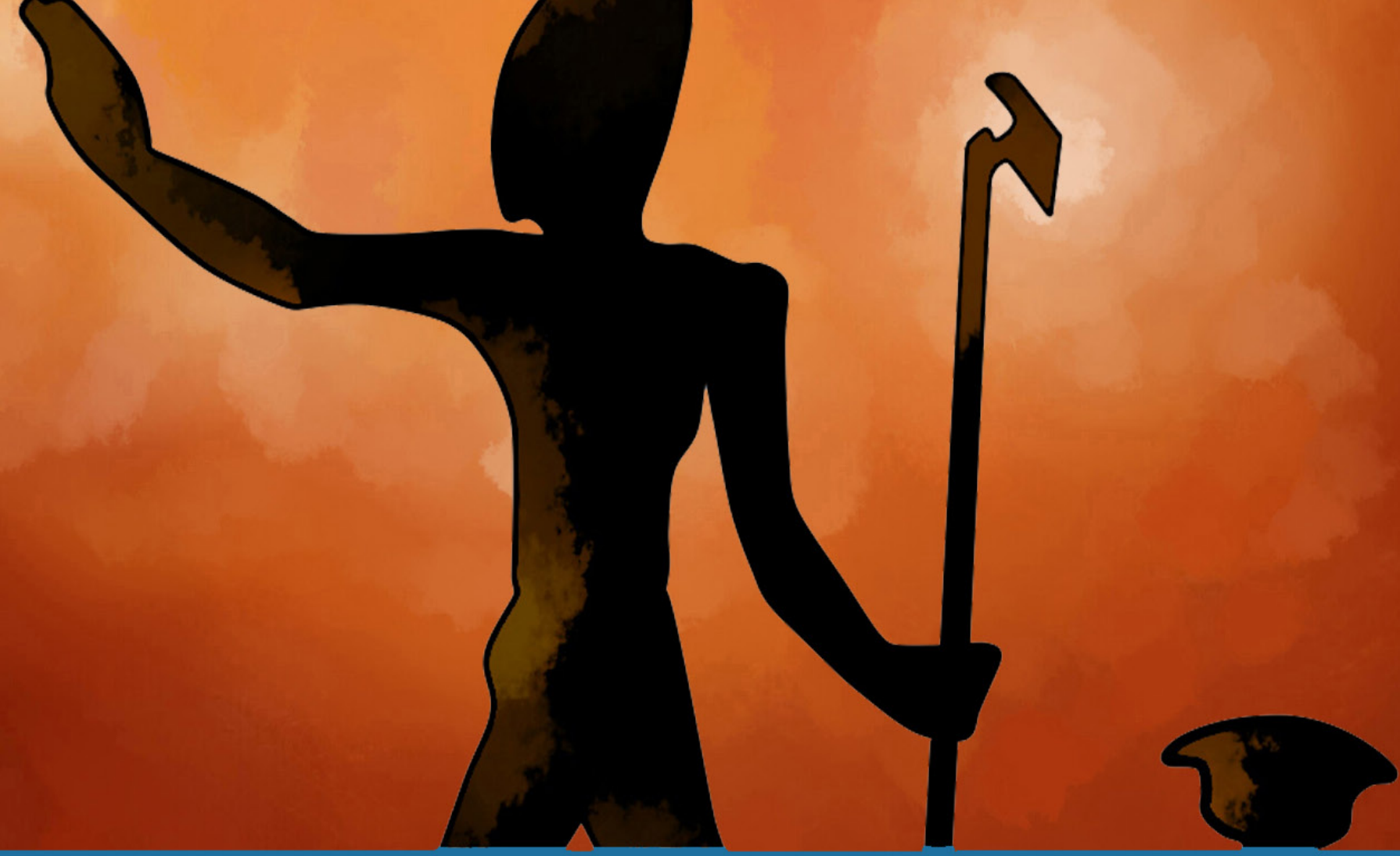

GepOA

centro de estudios de

próximo oriente y la

antigüedad tardía 